\title{
OPTIMIZATION OF BLOCK-COIL DIPOLES FOR HADRON COLLIDERS ${ }^{\ni}$
}

\author{
C. Battle, N. Diaczenko, T. Elliott, D. Gross, E. Hill, W. Henchel, M. Johnson, P. McIntyre\#, \\ A. Ravello, A. Sattarov, R. Soika, D. Wind, Texas A\&M University, College Station, TX \\ R. Gaedke, Trinity University, San Antonio, TX
}

\begin{abstract}
A first model dipole is being built for a 16 Tesla blockcoil dipole for future hadron colliders. The design uses stress management: a support matrix that intercepts Lorentz stress between successive sections of the coil and bypasses it to prevent strain degradation of the superconductors and insulation. The block-coil methodology has also been used to design dipoles for 12 Tesla and 15 Tesla, in which the amount of superconductor is minimized by cabling copper stabilizer strands with superconductor strands. The 12 Tesla block-coil dipole requires only one-fifth as much superconductor as does a 12 Tesla $\cos \theta$ dipole that is being developed elsewhere.
\end{abstract}

\section{INTRODUCTION}

The technology of superconducting dipoles determines the cost and performance of future hadron colliders. The field strength determines the relation between energy and circumference; the field quality and provisions for beam stability and synchrotron radiation determine the luminosity and lifetime of the colliding beams. Over the past several years much work has been done to relate the several requirements of a high-luminosity collider to the parameters of its magnets. A first example is synchrotron radiation. It was once thought that field strength beyond $\sim 10$ Tesla would create a problem from the heat deposited by synchrotron radiation in the cryogenic magnet. it is now realized that synchrotron radiation damping at high field strength can damp beam size and improve luminosity, and schema have been conceived (one presented below) whereby the synchrotron radiation can be absorbed at a higher temperature within the dipoles so that its refrigeration impact is reduced.

A second example is aperture. It was once thought that an aperture radius of at least $2.5 \mathrm{~cm}$ was necessary to have acceptable growth times for single-beam and mode coupling instabilities. Several schema have been developed recently whereby such instabilities can be damped within a single turn, so that apertures as small as $1 \mathrm{~cm}$ can support stable beams. With presently available superconductors, the coil for a high-field dipole is thick compared to its inner radius, so that reducing aperture has the potential to dramatically reduce magnet cost.

A third example is the impact of various multipoles upon beam growth mechanisms in a high-luminosity collider.
With NbTi superconductor, it was possible to make strands with extremely small filament diameter (few $\mu \mathrm{m}$ ) so that multipoles produced by persistent currents at injection energy were suppressed. NbTi cannot support fields higher than $\sim 9$ Tesla, and the superconductors that are used at higher fields (today $\mathrm{Nb}_{3} \mathrm{Sn}$, in the future $\mathrm{Bi}-2212$ ) currently have very large filament diameter $(>50 \mu \mathrm{m})$ if fabrication is optimized for high current density.

\section{BLOCK COIL DESIGN STRATEGY}

We are developing a new approach to dipole design, in which the coils are configured in rectangular blocks instead of the $\cos \theta$ geometry used in most superconducting dipoles to date. We are currently building a 16 Tesla dual dipole embodying this approach. We report here the results of recent work to optimize the block coil approach in the placement and composition of superconducting coil elements for 12 Tesla and 15 Tesla versions of the same design. The 12 Tesla design is shown in Figure 1.

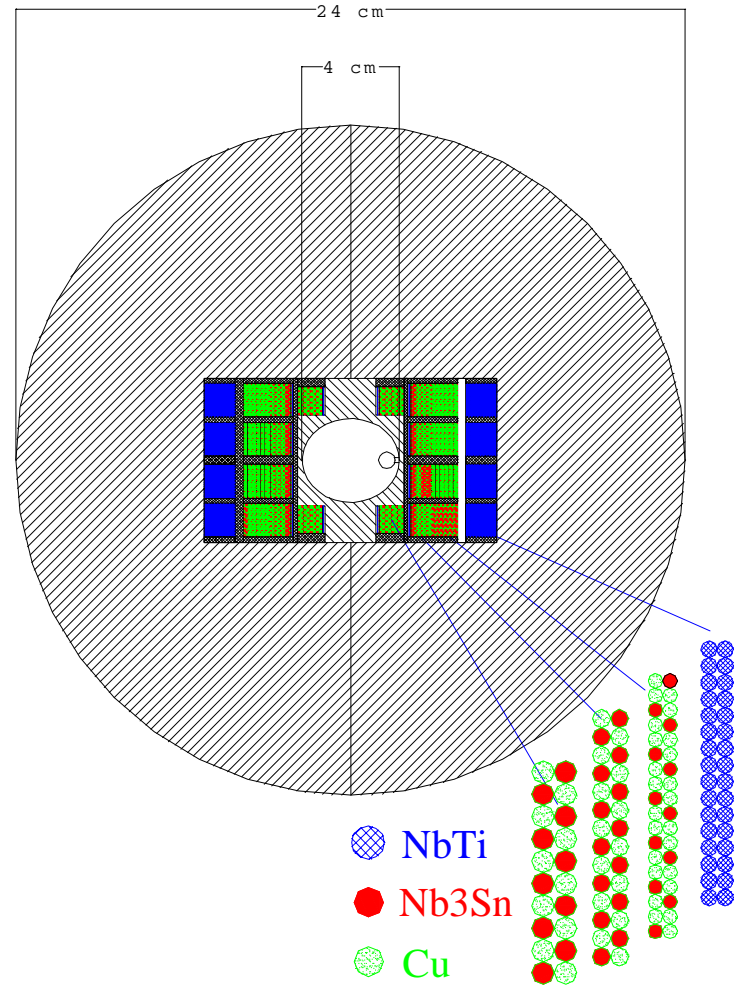

Figure 1. 12 Tesla block-coil dipole: optimized $\mathrm{Nb}_{3} \mathrm{Sn}, \mathrm{NbTi}$.

\footnotetext{
${ }^{\ni}$ Work supported in part by U.S. Dept. of Energy grant \#\#DE-FG03-95ER40924.

\# Email: p-mcintyre@physics.tamu.edu
} 


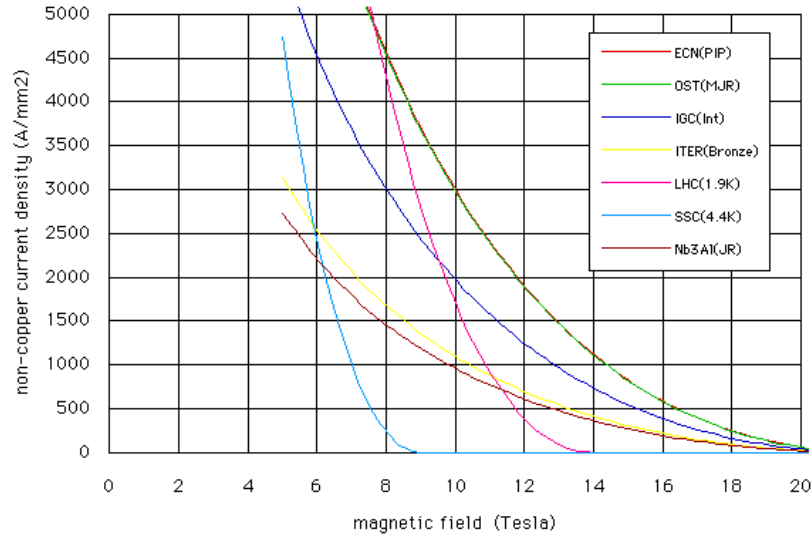

Figure 2. $\mathrm{j}_{\mathrm{c}}$ vs. B for various superconductors.

The aperture can be optimized to the application. The block-coil geometry is intrinsically scalable: the coil structure can be scaled to optimum for the aperture that is required for collider application, and also for the cable current that is best for quench protection (typically $\sim 10$ $\mathrm{kA})$. This feature is distinct from $\cos \theta$ coil geometry, in which the arch configuration of high-current cables makes it problematic to optimize with less than a $2.5 \mathrm{~cm}$ aperture radius. The design in Figure 1 has an aperture of $3.5 \mathrm{~cm}$ horizontal, $3 \mathrm{~cm}$ vertical.

Block-coils enable stress management. For field strength beyond $\sim 12$ Tesla, the stress in the coils of a superconducting dipole exceeds $100 \mathrm{MPa}$, at which point superconductors like $\mathrm{Nb}_{3} \mathrm{Sn}$ and Bi-2212 experience degradation of critical current capability. We devised a strategy [1] in which the stresses from the magnetic field acting upon the inner portions of a coil are intercepted by a support matrix that is distributed within the block-coil assembly. In this way the stress on superconducting elements can be limited to $<70 \mathrm{MPa}$ everywhere in the coil, even for fields as great as 20 Tesla.

Block-coil geometry suppresses persistent-current multipoles. The steel flux return in a block-coil dipole forms a planar boundary above and below the coil region. At injection field strength the steel is unsaturated, and this planar boundary condition suppresses multipoles arising from persistent current magnetization within the coil. For the 16 Tesla design of Ref. 1, the sextupole is suppressed by a factor 10 at injection.

Block-coil assemblies are easy to build. Each coil is a racetrack pancake coil. The coils are wound with simple fixturing and tight dimensional control can be achieved. While the center pancake coils must be bent out-of-plane to accommodate the beam tube, we have succeeded in winding them as planar coils and bending the completed coils in as a unit.

\section{CONDUCTOR OPTIMIZATION}

Figure 2 shows the magnetic field dependence of the critical current density $\mathrm{j}_{\mathrm{c}}$ in various superconductors. In a high-field dipole the coil is arranged in several segment: the inner segments are immersed in higher field

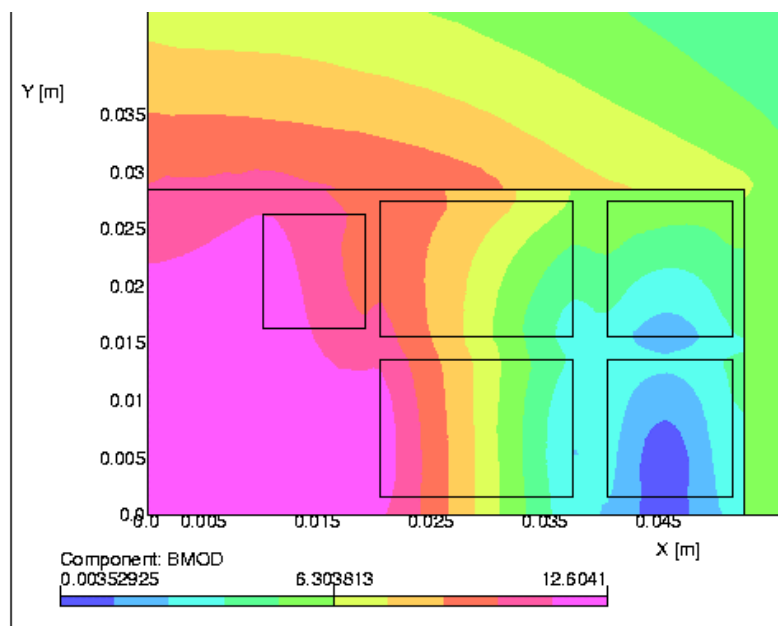

Figure 3. Field strength (modulus) in $12 \mathrm{~T}$ dipole.

and so can carry less current density than the outer segments. At the same time the magnet must be stable against quench. There must be sufficient copper within each cable that a quench can safely propagate throughout the coil without creating a damaging "hot spot" where it began. Current-day quench protection strategies for high-field dipoles typically require that the copper/superconductor ratio be sufficient that the current density $\mathrm{j}_{\mathrm{Cu}}$ in the copper during quench not exceed $\sim 1,500 \mathrm{~A} / \mathrm{mm}^{2}$.

Figure 3 shows the field distribution in the block-coil dipole of Figure 1. The contours of constant field strength are arranged in nearly vertical planes, so that the conductor in successive coil segments can be graded in $\mathrm{Cu}: \mathrm{SC}$ ratio to maintain this stability requirement. The conventional way to grade conductor in a high-field magnet is to incorporate the required fraction of copper into the billet with the superconductor so that it is drawn together with the superconducting filaments to form strands. There are two reasons why this approach is problematic at high field. First, it is expensive: the main cost in making superconducting strand is the complex processing to stack, draw, and heat treat the multi-filament strands. Adding copper proportionally increases the amount of this expensive commodity that is needed. The second reason is that the copper is actually degraded in its cold conductivity by the strand processing. The residual resistivity ratio $\mathrm{RRR}=$ $\rho_{300 \mathrm{~K}} / \rho_{4 \mathrm{~K}}$ of pure copper is $\sim 500$, that of the copper in superconducting strand is typically $\sim 50$.

We choose instead to provide most of the copper in the form of pure $\mathrm{Cu}$ strands that are cabled along with the superconducting strands into the Rutherford cable for each coil. In this way the $\mathrm{Cu}$ strands add very little cost, and the material cost arises mainly from that of the superconducting strands.

Indeed we take this approach one step further in the 12 Tesla design. The outermost coil segment has a maximum field of 6 Tesla. We use NbTi cable for this coil. As seen from Figure 2, its current density at this field is equal to that of $\mathrm{Nb}_{3} \mathrm{Sn}$ and it is much less expensive. 
Table 1. Optimized conductors for the coil segments of the $12 \mathrm{~T}$ and $15 \mathrm{~T}$ block-coil dipoles.

\begin{tabular}{|c|l|l|l|l|l|l|}
\hline Magnet, coil & superconductor & $\begin{array}{l}\mathrm{B}_{\max } \\
\text { Tesla }\end{array}$ & $\begin{array}{l}\text { jsc } \\
\mathrm{A} / \mathrm{mm}^{2}\end{array}$ & $\mathrm{Cu}: \mathrm{SC}$ & $\begin{array}{l}\text { SC strand area } \\
\left(\mathrm{cm}^{2}\right)\end{array}$ & $\begin{array}{l}\text { Total } \mathrm{Nb}_{3} \text { Sn strand } \\
\text { area in dipole }\left(\mathrm{cm}^{2}\right)\end{array}$ \\
\hline 12 Tesla block-coil & & & & & & \\
\hline sextupole, inner & $\mathrm{Nb}_{3} \mathrm{Sn}$ & $12.5,12.2$ & 2,000 & 1.4 & $1.25,2.36$ & 5.6 \\
\hline middle & $\mathrm{Nb}_{3} \mathrm{Sn}$ & 9 & 3,600 & 2.4 & 1.97 & \\
\hline outer & $\mathrm{NbTi}$ & 6 & & & 3.94 & \\
\hline 12 Tesla cos $\boldsymbol{\theta}$ & $\mathrm{Nb}_{3} \mathrm{Sn}$ & & & & & 28 \\
\hline
\end{tabular}

The impact of this approach is evident in Table 1, which summarizes the maximum field, the $\mathrm{Cu}: \mathrm{SC}$ ratio required for stability, and the cross-sectional area of superconducting strand required for the 12 Tesla design of Figure 1 and a 12 Tesla ( $5 \mathrm{~cm}$ aperture) $\cos \theta$ dipole being developed at Fermilab[2]. It had once been thought that block-coil designs were intrinsically less current-efficient than $\cos \theta$ designs. This is demonstrably not the case.

\section{FIELD QUALITY}

The field quality in a block-coil dipole is dominated at injection field by the boundary condition of the steel flux return, and at high field by the conductor placement. We
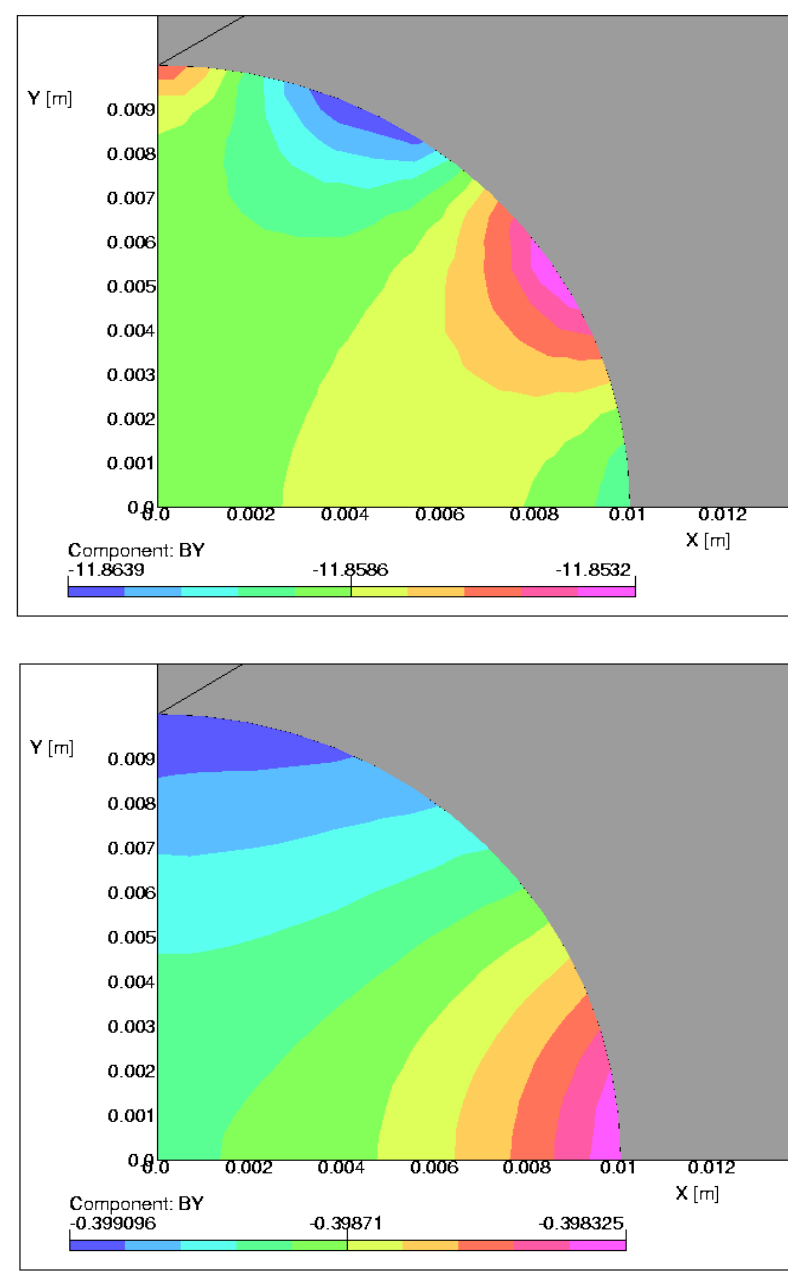

Figure 4. Field distribution at center of $12 \mathrm{~T}$ dipole: a) injection; b) max field. provide for correction of the multipoles that arise from saturation by placing a sextupole correction winding above and below the beam tube region, as shown in Figure 1. The correction winding is designed to be off at injection, and carries the same current as the main windings at maximum field. Figure 4 shows the calculated field distribution within a radius of $1 \mathrm{~cm}$, at injection and maximum field. In each case the sextupole is about $10^{-4} \mathrm{~cm}^{-2}$. This current programming enables us to suppress the dominant multipoles over the entire range of operation.

\section{SYNCHROTRON RADIATION AND CRYOGENICS}

The synchrotron radiation in a high-field dipole can be a pacing consideration, both for effective cooling of the dipoles and for the overall refrigeration budget in a collider. We contemplate the incorporation of a cooling channel within the beam tube, internally cooled by either liquid neon $\left(\sim 30^{\circ} \mathrm{K}\right)$ or liquid nitrogen $\left(80^{\circ} \mathrm{K}\right)$. The tube is shown in Figure 1, located in the mid-plane to the radial outside in the collider so that it forms the first wall upon which synchrotron radiation is absorbed. By taking the heat of the synchrotron radiation at such elevated temperature, the refrigeration needed to remove it to ambient is dramatically reduced. We have simulated such a conductor geometry in TBCI and SUPERFISH; no problematic higher-order modes are driven. Gas molecules that are desorbed from the tube walls by the synchrotron radiation should be trapped on the $\left(4^{\circ} \mathrm{K}\right)$ walls of the beam tube after a traversal of the vacuum space.

\section{CONCLUSIONS}

The block-coil dipole provides an effective basis for optimizing the design of a high-field dipole to make most effective use of superconductor. The separation of stabilizing copper into separate strands within each cable offers a dramatic reduction in conductor cost. The impact of this separation upon stability against micro-quenches and upon AC losses must be studied to determine whether it is consistent with all of the many requirements upon the dipoles of a hadron collider.

[1] N. Diaczenko, Stress management in high-field dipoles, Proc. 1997 Particle Accel. Conf. Vancouver, 1997.

[2] T. Arcan et al., "Conceptural design of the Fermilab $\mathrm{Nb}_{3} \mathrm{Sn}$ high-field dipole model", this proceedings. 